\title{
A Complete Map of Conditional Knowledge Bases in Different Normal Forms and Their Induced System P Inference Relations Over Small Signatures
}

\author{
Christoph Beierle \\ FernUniversität in Hagen \\ Hagen, Germany \\ christoph.beierle@fernuni-hagen.de
}

\author{
Jonas Haldimann \\ FernUniversität in Hagen \\ Hagen, Germany \\ jonas.haldimann@fernuni-hagen.de
}

\author{
Steven Kutsch \\ FernUniversität in Hagen \\ Hagen, Germany \\ steven.kutsch@fernuni-hagen.de
}

\begin{abstract}
Conditional knowledge bases consisting of qualitative conditionals play a predominant role in knowledge representation and reasoning. In this paper, we develop a full map of all consistent conditional knowledge bases over a small signature in different normal forms. We introduce two new normal forms that take the induced system $P$ inference relation into account, the system $P$ normal form (SPNF) and the renaming $S P N F$ $(\rho \mathrm{SPNF})$ considering additionally renamings of the underlying signature. For a two-element signature, we systematically generate and compare all consistent knowledge bases in ANF, RANF, SPNF, and their renaming counterparts, as well as all complete system $P$ inference relations induced by conditional knowledge bases.
\end{abstract}

\section{Introduction}

The richness of different syntactic expressions as in, e.g., first-order logic, is desirable for expressing things from different points of view or for meeting linguistic preferences of a user. On the other hand, for comparing the semantics of syntactic descriptions or for automatically processing them, normal forms may provide considerable advantages (Robinson 1965; Robinson and Voronkov 2001). In this paper, we investigate representations of knowledge bases consisting of qualitative conditionals which play a predominant role in knowledge representation and reasoning (e.g. (Adams 1975; Kraus, Lehmann, and Magidor 1990; Dubois and Prade 1994; Goldszmidt and Pearl 1996; Benferhat, Dubois, and Prade 1999; Kern-Isberner 2001)). We develop a full map of all consistent conditional knowledge bases over a small signature in different normal forms. The normal forms take various syntactical and semantical aspects into account, ranging from classical canonical disjunctive normal forms (CDNF) in the underlying propositional language to the full system $\mathrm{P}$ inference relation induced by a knowledge base.

Besides CDNF, we consider the antecedent normal form (ANF) (Beierle and Kutsch 2019a) and the reduced antecedent normal form (RANF) (Beierle and Haldimann 2020a), as well as their variants that also take renamings of the underlying signature into account ( $\rho \mathrm{ANF}, \rho \mathrm{RANF}$ ). Furthermore, we introduce new normal forms for conditional knowledge bases that take their induced system P (Lehmann

Copyright @ 2021 by the authors. All rights reserved. and Magidor 1992) inference relation into account, the system $P$ normal form (SPNF) and its renaming counterpart ( $\rho$ SPNF). Focussing on the signature $\Sigma_{a b}=\{a, b\}$, our investigations show, for instance, that every of the at least 555.135.087 different consistent $\Sigma_{a b}$-knowledge bases $\mathcal{R}$ in CDNF can be transformed into a unique $\mathcal{R}^{\prime}$ where $\mathcal{R}^{\prime}$ is one of $262 \Sigma_{a b}$-knowledge bases in $\rho$ SPNF and $\mathcal{R}$ and $\mathcal{R}^{\prime}$ are inferentially renaming equivalent with respect to system $\mathrm{P}$. In summary, the main contributions of this paper are:

- Presentation of a sequence of normal forms for conditional knowledge bases with respect to different syntactic and semantic equivalences.

- Introduction of the new normal forms SPNF and $\rho$ SPNF.

- Systematic generation and comparison of all consistent knowledge bases over $\Sigma_{a b}$ in ANF, RANF, SPNF and their renaming counterparts.

- Generation of all complete system $\mathrm{P}$ inference relations induced by conditional knowledge bases over $\Sigma_{a b}$.

\section{Background: Conditional Logic}

Let $\mathcal{L}(\Sigma)$ be the propositional language over a finite signature $\Sigma$. We call a signature $\Sigma$ with a linear ordering $\lessdot$ an ordered signature and denote it by $(\Sigma, \lessdot)$. The language may be denoted by $\mathcal{L}$ if the signature is clear from context. The formulas of $\mathcal{L}$ will be denoted by letters $A, B, C, \ldots$ We write $A B$ for $A \wedge B$ and $\bar{A}$ for $\neg A$. We identify the set of all complete conjunctions over $\Sigma$ with the set $\Omega$ of possible worlds over $\mathcal{L}$. For $\omega \in \Omega$ and $A \in \mathcal{L}, \omega \models A$ means that $A$ holds in $\omega$. The set of worlds satisfying $A$ is $\Omega_{A}=\{\omega \mid \omega \models A\}$. Two formulas $A, B$ are equivalent, denoted as $A \equiv B$, if $\Omega_{A}=\Omega_{B}$.

By introducing a new binary operator $\mid$, we obtain the set $(\mathcal{L} \mid \mathcal{L})_{\Sigma}=\{(B \mid A) \mid A, B \in \mathcal{L}(\Sigma)\}$ of conditionals over $\mathcal{L}(\Sigma)$. Again, $\Sigma$ may be omitted. As semantics for conditionals, we use ordinal conditional functions $(O C F)$, also called ranking functions, first introduced (in a more general form) in (Spohn 1988). An OCF is a function $\kappa: \Omega \rightarrow \mathbb{N} \cup\{\infty\}$ expressing degrees of plausibility of possible worlds where a lower degree denotes "less surprising". At least one world must be regarded as being normal; therefore, $\kappa(\omega)=0$ for at least one $\omega \in \Omega$. Each $\kappa$ uniquely extends to a function mapping formulas to $\mathbb{N} \cup\{\infty\}$ given by $\kappa(A)=\min \{\kappa(\omega) \mid$ 
$\omega \models A\}$ where $\min \emptyset=\infty$. An OCF $\kappa$ accepts a conditional $(B \mid A)$, written $\kappa \models(B \mid A)$, if the verification of the conditional is less surprising than its falsification, i.e., if $\kappa(A B)<\kappa(A \bar{B})$; equivalently, $\kappa \models(B \mid A)$ iff for every $\omega^{\prime} \in \Omega_{A \bar{B}}$ there is $\omega \in \Omega_{A B}$ with $\kappa(\omega)<\kappa\left(\omega^{\prime}\right)$. A conditional $(B \mid A)$ is trivial if it is self-fulfilling $(A \models B)$ or contradictory $(A \models \bar{B})$; for avoiding cumbersome and noninformative case distinctions, we will restrict our attention to non-trivial conditionals. A finite, non-empty set $\mathcal{R} \subseteq(\mathcal{L} \mid \mathcal{L})$ of non-trivial conditionals is called a knowledge base. An OCF $\kappa$ accepts a knowledge base $\mathcal{R}$ if $\kappa$ accepts all conditionals in $\mathcal{R}$, and $\mathcal{R}$ is consistent if an OCF accepting $\mathcal{R}$ exists. We use $\diamond$ to denote an inconsistent knowledge base. $\operatorname{Mod}(\mathcal{R})$ denotes the set of all OCFs $\kappa$ accepting $\mathcal{R}$. Two knowledge bases $\mathcal{R}, \mathcal{R}^{\prime}$ are model equivalent, denoted by $\mathcal{R} \equiv{ }_{\text {mod }} \mathcal{R}^{\prime}$, if $\operatorname{Mod}(\mathcal{R})=\operatorname{Mod}\left(\mathcal{R}^{\prime}\right)$. Correspondingly, we say that $(B \mid A)$ and $\left(B^{\prime} \mid A^{\prime}\right)$ are equivalent, denoted by $(B \mid A) \equiv\left(B^{\prime} \mid A^{\prime}\right)$, if $A \equiv A^{\prime}$ and $A B \equiv A^{\prime} B^{\prime}$.

System $P$ (Lehmann and Magidor 1992) allows reasoning about conditional knowledge bases and consists of the following six axioms:

$\begin{array}{lr}\text { (RE) } & \text { for all } A \in \mathcal{L} \text { it holds that } A \sim A \\ (L L E) & A \equiv B \text { and } A \sim C \text { imply } B \sim C \\ (R W) & B=C \text { and } A \sim B \text { imply } A \sim C \\ (A N D) & A \sim B \text { and } A \sim C \text { imply } A \sim B \wedge C \\ (O R) & A \sim C \text { and } B \sim C \text { imply }(A \vee B) \sim C \\ (C M) & A \sim B \text { and } A \sim C \text { imply } A \wedge B \sim C\end{array}$

If $B$ can be derived from $A$ using the knowledge base $\mathcal{R}$ by applying the rules in system $\mathrm{P}$, we denote this by $A \sim_{\mathcal{R}}^{p} B$. It has been shown (see (Adams 1975; Pearl 1988; Dubois and Prade 1994; Lehmann and Magidor 1992)) that system $P$ inference coincides with p-entailment (Goldszmidt and Pearl 1996) where $A$ p-entails $B$ in the context of $\mathcal{R}$, denoted as $A \sim_{\mathcal{R}} B$, iff all models of $\mathcal{R}$ accept $(B \mid A)$.

\section{Normal Form Conditionals and Renamings}

For developing a method for the systematic generation of knowledge bases over a given signature $\Sigma$, we first observe that the set of syntactically different conditionals and also the set of different knowledge bases over $\Sigma$ is infinite because $\mathcal{L}=\mathcal{L}(\Sigma)$ is infinite. In order to obtain a finite set, we abstract from the rich syntactic variants of the underlying propositional language $\mathcal{L}$ and represent each formula $A \in \mathcal{L}$ uniquely by its set $\Omega_{A}$ of satisfying worlds, called canonical disjunctive normal form (CDNF) of $A$.

Example 1. Let $\mathcal{R}_{81}=\{(\bar{a} \mid b),(b \mid a \vee b)\}$. Using the CDNF for propositional formulas, we obtain $\mathcal{R}_{81}^{\prime}=$ $\{(\{\bar{a} b, \bar{a} \bar{b}\} \mid\{a b, \bar{a} b\}),(\{a b, \bar{a} b\} \mid\{a b, a \bar{b}, \bar{a} b\})\}$.

Example 2. Using CDNF for $\mathcal{R}_{935}=\{(\bar{a} \mid b),(b \mid a \vee b),(\bar{a} \vee b$ $\mid a \vee \bar{b})\}$ we obtain $\mathcal{R}_{935}^{\prime}=\{(\{\bar{a} b, \bar{a} \bar{b}\} \mid\{a b, \bar{a} b\}),(\{a b, \bar{a} b\}$ $\mid\{a b, a \bar{b}, \bar{a} b\}),(\{a b, \bar{a} b, \bar{a} \bar{b}\} \mid\{a b, a \bar{b}, \bar{a} \bar{b}\})\}$.

We can further simplify the CDNF of conditionals by using normal form conditionals (Beierle and Kutsch 2019b). In the following proposition, the two conditions $B \varsubsetneqq A$ and $B \neq \emptyset$ ensure the falsifiability and the verifiability of a conditional $(B \mid A)$, thereby excluding any trivial conditional.
Proposition $1(N F C(\Sigma)$ (Beierle and Kutsch 2019b)). For $N F C(\Sigma)=\left\{(B \mid A) \mid A \subseteq \Omega_{\Sigma}, B \varsubsetneqq A, B \neq \emptyset\right\}$, the set of normal form conditionals over $\Sigma$, the following holds:

(nontrivial) $N F C(\Sigma)$ does not contain any trivial conditional.

(complete) For every nontrivial conditional over $\Sigma$ there is an equivalent conditional in $N F C(\Sigma)$.

(minimal) All conditionals in $N F C(\Sigma)$ are pairwise nonequivalent.

Example 3. Replacing every conditional in $\mathcal{R}_{935}^{\prime}$ by its equivalent normal form conditional yields $\mathcal{R}_{935}^{\prime \prime}=\{(\{\bar{a} b\}$ $\mid\{a b, \bar{a} b\}),(\{a b, \bar{a} b\} \mid\{a b, a \bar{b}, \bar{a} b\}),(\{a b, \bar{a} \bar{b}\} \mid\{a b, a \bar{b}, \bar{a} \bar{b}\})\}$.

Normal form conditionals are sufficient to represent every knowledge base up to equivalence induced by system $\mathrm{P}$.

Definition 1. Two knowledge bases $\mathcal{R}, \mathcal{R}^{\prime}$ are inferentially equivalent (with respect to system $P$ ), denoted by $\mathcal{R} \stackrel{p}{\sim} \mathcal{R}^{\prime}$, if $A \sim_{\mathcal{R}}^{p} B$ holds if and only if $A \sim_{\mathcal{R}^{\prime}}^{p} B$ for all formulas $A, B$. The following is well-known (Goldszmidt and Pearl 1996):

Proposition 2. Let $\mathcal{R}, \mathcal{R}^{\prime}$ be knowledge bases. Then

$$
\mathcal{R} \equiv_{\text {mod }} \mathcal{R}^{\prime} \text { if and only if } \mathcal{R} \stackrel{p}{\sim} \mathcal{R}^{\prime}
$$

Proposition 3. Let $\mathcal{R}, \mathcal{R}^{\prime}$ be knowledge bases such that $\mathcal{R}$ is consistent and $\mathcal{R}^{\prime}$ is obtained from $\mathcal{R}$ by replacing every conditional $(B \mid A)$ in $\mathcal{R}$ by $\left(B^{\prime} \mid A^{\prime}\right)$ where $A^{\prime}$ is the CDNF of $A$ and $B^{\prime}$ is the CDNF of $A B$. Then $\mathcal{R} \stackrel{p}{\sim} \mathcal{R}^{\prime}$.

Proof. Model equivalence of $(B \mid A)$ with $(A B \mid A)$ and its CDNF ensures $\mathcal{R} \equiv{ }_{\bmod } \mathcal{R}^{\prime}$, thus $\mathcal{R} \stackrel{p}{\sim} \mathcal{R}^{\prime}$ due to (1).

Apart from avoiding generating syntactic variants of conditionals and of knowledge bases, we also want to take symmetries into account that are induced by isomorphisms on the underlying signature. For a signature $\Sigma$, a function $\rho: \Sigma \rightarrow \Sigma^{\prime}$ is a renaming if $\rho$ is a bijection. For instance, the function $\rho_{a b}$ with $\rho_{a b}(a)=b$ and $\rho_{a b}(b)=a$ is a renaming for $\Sigma_{a b}$. As usual, $\rho$ is extended canonically to worlds, formulas, conditionals, knowledge bases, and to sets thereof.

Definition $2(\simeq)$. Let $X, X^{\prime}$ be two signatures, worlds, formulas, knowledge bases, sets, or relations over one of these items. We say that $X$ and $X^{\prime}$ are isomorphic with respect to signature renamings, denoted by $X \simeq X^{\prime}$, if there exists a renaming $\rho$ such that $\rho(X)=X^{\prime}$.

For a set $M, m \in M$, and an equivalence relation $\equiv$ on $M$, the set of equivalence classes induced by $\equiv$ is denoted by $[M]_{/ \equiv}$, and the unique equivalence class containing $m$

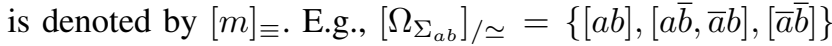
are the three equivalence classes of worlds over $\Sigma_{a b}$, and we have $[(a b \mid a b \vee a \bar{b})]_{\simeq}=[(a b \mid a b \vee \bar{a} b)]_{\simeq \text {. }}$.

Given a signature $\Sigma$ with linear ordering $\lessdot$, in (Beierle and Haldimann 2020a) an induced linear ordering $\prec$ on $N F C(\Sigma)$ is defined. While the details of this ordering are not needed here, for an illustration, Table 1 shows some of the conditionals in $\operatorname{NFC}\left(\Sigma_{a b}\right)$ and their induced ordering $\prec$. The set $\operatorname{NFC}\left(\Sigma_{a b}\right)$ contains 50 conditionals, and $\left[\operatorname{NFC}\left(\Sigma_{a b}\right)\right] / \simeq$ has 31 equivalence classes; 19 of these 


\begin{tabular}{lll}
\hline class & \multicolumn{1}{c}{ first conditional } & second conditional \\
\hline$[01]$ & $r_{01}:(\{a b\} \mid\{a b, a \bar{b}\})$ & $r_{02}:(\{a b\} \mid\{a b, \bar{a} b\})$ \\
{$[02]$} & $r_{03}:(\{a \bar{b}\} \mid\{a b, a \bar{b}\})$ & $r_{04}:(\{\bar{a} b\} \mid\{a b, \bar{a} b\})$ \\
{$[03]$} & $r_{05}:(\{a b\} \mid\{a b, \bar{a} \bar{b}\})$ & \\
{$[04]$} & $r_{06}:(\{\bar{a} \bar{b}\} \mid\{a b, \bar{a} \bar{b}\})$ & \\
{$[05]$} & $r_{07}:(\{a \bar{b}\} \mid\{a \bar{b}, \bar{a} b\})$ & $r_{08}:(\{\bar{a} b\} \mid\{a \bar{b}, \bar{a} b\})$ \\
$\ldots$ & $\ldots$ & $\ldots$ \\
\hline
\end{tabular}

Table 1: The first eight of the conditionals $r_{01} \prec \ldots \prec r_{50}$ in $\operatorname{NFC}\left(\Sigma_{a b}\right)$ given in CDNF for $\Sigma_{a b}=\{a, b\}$, and their equivalence classes $[01], \ldots,[31]$ induced by signature renamings

classes contain two conditionals, while the other 12 classes are singletons. The $\prec-$ minimal conditional in each equivalence class is the canonical representative of that class, called canonical normal form conditional.

Observation $1\left(\operatorname{NFC}\left(\Sigma_{a b}\right)\right)$. The algorithm GenKB (Beierle and Kutsch 2019b, Algorithm 1) generates systematically consistent knowledge bases over a given signature. It also takes renamings into account to the extent that the $\prec$-least conditional in each generated knowledge base is a canonical normal form conditional. Our implementation of GenKB reveals that there are 555.135.087 such knowledge bases over $\Sigma_{a b}$. Each knowledge base contains between 1 and 25 conditionals. The number of knowledge bases of each size varies vastly. For example, GenKBgenerates only 24 knowledge bases with 25 conditionals but 88.986 .856 knowledge bases with 13 conditionals.

Observation $2\left(\operatorname{NFC}\left(\Sigma_{a b}\right)\right)$. We modified the algorithm $G e n K B$ in such a way that more renamings are taken into account. Instead of 555.135.087 knowledge bases, the implementation of this refined algorithm generates 364.304.482 $\Sigma_{a b}$-knowledge bases, still capturing all consistent knowledge bases over $\Sigma_{a b}$ up to inferential equivalence and renaming. Since the results differ from the knowledge bases generated by $G e n K B$ only by not generating some renamings, the maximal number of conditionals in a knowledge base stays the same, i.e., 25 conditionals.

\section{Antecedent Normal Form}

The idea of the notion of antecedentwise equivalence is to take into account the set of conditionals having the same (or propositionally equivalent) antecedent when comparing to knowledge bases.

Definition $3\left(A n t(\mathcal{R}), \mathcal{R}_{\mid A}\right.$, ANF (Beierle and Kutsch 2019a)). Let $\mathcal{R}$ be a knowledge base.

- $\operatorname{Ant}(\mathcal{R})=\{A \mid(B \mid A) \in \mathcal{R}\}$ are the antecedents of $\mathcal{R}$.

- For $A \in \operatorname{Ant}(\mathcal{R})$, the set $\mathcal{R}_{\mid A}=\left\{\left(B^{\prime} \mid A^{\prime}\right) \mid\left(B^{\prime} \mid A^{\prime}\right) \in\right.$ $\mathcal{R}$ and $\left.A \equiv A^{\prime}\right\}$ is the set of $A$-conditionals in $\mathcal{R}$.

- $\mathcal{R}$ is in antecedent normal form (ANF) if either $\mathcal{R}$ is inconsistent and $\mathcal{R}=\diamond$, or $\mathcal{R}$ is consistent, does not contain any self-fulfilling conditional, contains only conditionals of the form $(A B \mid A)$, and $\left|\mathcal{R}_{\mid A}\right|=1$ for all $A \in \operatorname{Ant}(\mathcal{R})$.

Definition 4 ( $\ll_{a e}$, equivalence $\equiv_{a e}$ (Beierle and Kutsch 2019a)). Let $\mathcal{R}, \mathcal{R}^{\prime}$ be knowledge bases.
- $\mathcal{R}$ is an antecedentwise equivalent sub-knowledge base of $\mathcal{R}^{\prime}$, denoted by $\mathcal{R} \ll_{a e} \mathcal{R}^{\prime}$, if for every $A \in \operatorname{Ant}(\mathcal{R})$ such that $\mathcal{R}_{\mid A}$ is not self-fulfilling there is an $A^{\prime} \in \operatorname{Ant}\left(\mathcal{R}^{\prime}\right)$ with $\mathcal{R}_{\mid A} \equiv_{\text {mod }} \mathcal{R}_{\mid A^{\prime}}^{\prime}$.

- $\mathcal{R}$ and $\mathcal{R}^{\prime}$ are strictly antecedentwise equivalent if $\mathcal{R} \ll_{a e}$ $\mathcal{R}^{\prime}$ and $\mathcal{R}^{\prime} \ll_{a e} \mathcal{R}$.

- $\mathcal{R}$ and $\mathcal{R}^{\prime}$ are antecedentwise equivalent, denoted by $\mathcal{R} \equiv{ }_{a e} \mathcal{R}^{\prime}$, if either both are inconsistent, or both are consistent and strictly antecedentwise equivalent.

Note that any two inconsistent knowledge bases are also antecedentwise equivalent according to Definition 4, e.g., $\{(b \mid a),(\bar{b} \mid b)\} \equiv_{a e}\{(b \mid b),(a \bar{a} \mid \top)\}$, enabling us to avoid cumbersome case distinctions when dealing with consistent and inconsistent knowledge bases.

Example 4. The two knowledge bases $\{(a \mid a \vee b),(b \mid a \vee b)\}$ and $\{(a b \mid a \vee b)\}$ are antecedentwise equivalent. $\mathcal{R}_{935}^{\prime \prime}$ from Example 3 is in ANF.

Antecedentwise equivalence ensures inferential equivalence.

Proposition 4. If $\mathcal{R}, \mathcal{R}^{\prime}$ are knowledge bases, $\mathcal{R} \equiv_{\text {ae }} \mathcal{R}^{\prime}$ implies $\mathcal{R} \stackrel{p}{\sim} \mathcal{R}^{\prime}$.

Proof. W.l.o.g. assume that $\mathcal{R}$ and $\mathcal{R}^{\prime}$ are consistent. From $\mathcal{R} \equiv_{a e} \mathcal{R}^{\prime}$, we get $\mathcal{R} \ll_{a e} \mathcal{R}^{\prime}$, and Definition 4 implies that there is a function $f: \operatorname{Ant}(\mathcal{R}) \rightarrow \operatorname{Ant}\left(\mathcal{R}^{\prime}\right)$ with $\mathcal{R}_{\mid A} \equiv_{\bmod } \mathcal{R}_{\mid f(A)}^{\prime}$ for each $A \in \operatorname{Ant}(\mathcal{R})$. Thus, $\mathcal{R}=\bigcup_{A \in A n t(\mathcal{R})} \mathcal{R}_{\mid A} \equiv_{\text {mod }} \bigcup_{A \in A n t(\mathcal{R})} \mathcal{R}_{\mid f(A)}^{\prime} \subseteq \mathcal{R}^{\prime}$ implies $\operatorname{Mod}\left(\mathcal{R}^{\prime}\right) \subseteq \operatorname{Mod}(\mathcal{R})$. Employing this argumentation in both directions, we get $\mathcal{R} \equiv_{\text {mod }} \mathcal{R}^{\prime}$ and therefore also $\mathcal{R} \stackrel{p}{\sim} \mathcal{R}^{\prime}$ due to Proposition 2.

A set of transformation rules can map every knowledge base into its uniquely determined ANF (Beierle and Kutsch 2019a). Furthermore, the algorithm $K B_{\text {gen }}^{a e}$ (Beierle and Kutsch 2019a, Algorithm 1) generates systematically consistent knowledge bases over a given signature in ANF using only normal form conditionals; it takes renaming into account as sketched in Observation 1 for the algorithm $G e n K B$, i.e., the ґ-least conditional in each generated knowledge base is a canonical normal form conditional.

Observation 3 (ANF). Our implementation of $K B_{\text {gen }}^{a e}$ shows that there are 758.808 knowledge bases over $\Sigma_{a b}$ in ANF such that the $\prec$-least conditional in the knowledge base is a canonical normal form conditional. Note that compared to the large numbers of 555.135.087 and 364.304.482 $\Sigma_{a b^{-}}$ knowledge bases generated by GenKB (cf. Observations 1 and 2), this huge reduction is achieved solely by employing the ANF of knowledge bases. As the ANF merges conditionals with the same antecedent, the knowledge bases also tend to be smaller. The 1.827 largest knowledge bases generated by $K B_{\text {gen }}^{a e}$ contain 11 conditionals.

The following proposition shows that $K B_{g e n}^{a e}$ captures all consistent knowledge bases over a given signature up to inferential equivalence and renamings. 
Proposition $5\left(K B_{\text {gen }}^{a e}\right)$. Applying $K B_{\text {gen }}^{a e}$ to an ordered signature $(\Sigma, \lessdot)$ terminates and returns a set of knowledge bases $\mathcal{K} \mathcal{B}$ for which the following holds:

( $\sim$ completeness) If $\mathcal{R}$ is a consistent $\Sigma$-knowledge base then there is $\mathcal{R}^{\prime} \in \mathcal{K B}$ and a signature renaming $\rho$ such that $\mathcal{R} \stackrel{p}{\sim} \rho\left(\mathcal{R}^{\prime}\right)$.

Proof. For every consistent $\Sigma$-knowledge base $\mathcal{R}$ there is $\mathcal{R}^{\prime} \in \mathcal{K} \mathcal{B}$ and a signature renaming $\rho$ such that $\mathcal{R} \equiv_{\text {mod }}$ $\rho\left(\mathcal{R}^{\prime}\right)$ (Beierle and Kutsch 2019a, Proposition 7), and thus $\mathcal{R} \stackrel{p}{\sim} \rho\left(\mathcal{R}^{\prime}\right)$ due to (1).

Observation 4 (ANF). The 758.808 knowledge bases over $\Sigma_{a b}$ generated by $K B_{g e n}^{a e}$ capture all consistent $\Sigma_{a b^{-}}$ knowledge bases up to renaming and inferential equivalence with respect to system $P$ inference.

\section{Knowledge Bases in RANF and in $\rho$ RANF}

While the antecedent normal form can compact conditional knowledge bases drastically, a knowledge base in ANF may still contain redundancies in form of conditionals that can be removed without changing the system $\mathrm{P}$ inference relation induced by the knowledge base.

Example 5. Consider $\mathcal{R}=\{(a b \mid a),(a b \mid b),(a b \mid a \vee b)\}$. The third conditional can be derived from the first two conditionals with system $\mathrm{P}$ axiom $(O R)$.

Proposition 6. Let $\mathcal{R}$ be a knowledge base and $A, B \in \mathcal{L}$ such that $A \sim_{\mathcal{R}}^{p} B$. Then $\sim_{\mathcal{R}}^{p}=\sim_{\mathcal{R} \cup\{(B \mid A)\}}^{p}$.

Proof. Let $(D \mid C) \in(\mathcal{L} \mid \mathcal{L})$ be a conditional. If $(D \mid C)$ has a system $\mathrm{P}$ derivation from $\mathcal{R}$, then it has a system $\mathrm{P}$ derivation from $\mathcal{R} \cup\{(B \mid A)\}$ because of the semi-monotonicity of system P. If $(D \mid C)$ can be derived from $\mathcal{R} \cup\{(B \mid A)\}$ with system $\mathrm{P}$, then it can be derived from $\mathcal{R}$ as well because $(B \mid A)$ can be derived from $\mathcal{R}$.

In Example 5, this implies that omitting the conditional $(a b \mid a \vee b)$ does not change the system $\mathrm{P}$ inference relation induced by the knowledge base. The reduced form is a knowledge base avoiding such redundancies.

Definition 5 (reduced form, RANF (Beierle and Haldimann 2020a)). Let $\mathcal{R}$ be a knowledge base.

- $\mathcal{R}$ is in reduced form (with respect to system $\mathrm{P}$ ) if there is no conditional $(B \mid A) \in \mathcal{R}$ such that $A \sim_{\mathcal{R} \backslash(B \mid A)}^{p} B$.

- $\mathcal{R}$ is in reduced antecedent normal form (RANF) if $\mathcal{R}$ is in ANF and in reduced form.

In (Beierle and Haldimann 2020a), a transformation system $\Theta^{r a}$ is provided such that every $\mathcal{R}^{\prime} \in \Theta^{r a}(\mathcal{R})$ is in RANF and model equivalent to $\mathcal{R}$.

The approaches to generate knowledge bases examined in Sec. 3 and 4 consider renamings to some extent, but the mentioned algorithms still generate some knowledge bases that are isomorphic with respect to signature renaming to another generated knowledge base. Also, the normal forms CDNF, NFC, ANF, and RANF do not take renamings into account. The renaming normal form avoids redundancies regarding renamings because any two knowledge bases in renaming normal form that are not equal are not isomorphic with respect to signature renaming. To define the renaming normal form, we will rely on the linear ordering $\prec$ on $N F C(\Sigma)$ (Beierle and Haldimann 2020a) sketched in Section 3, cf. Table 1 . We extend $\prec$ to an ordering on knowledge bases.

Definition $6(\preccurlyeq s e t)$. The lexicographic extension of the ordering $\preccurlyeq$ on $N F C(\Sigma)$ to strings over $N F C(\Sigma)$ is denoted by $\preccurlyeq$ lex. For knowledge bases $\mathcal{R}=\left\{r_{1}, \ldots, r_{n}\right\}$ and $\mathcal{R}^{\prime}=$ $\left\{r_{1}^{\prime}, \ldots, r_{n^{\prime}}^{\prime}\right\}$ over NFC $(\Sigma)$ with $r_{i} \prec r_{i+1}$ and $r_{j}^{\prime} \prec r_{j+1}^{\prime}$ the ordering $\preccurlyeq$ set is given by: $\mathcal{R} \preccurlyeq$ set $\mathcal{R}^{\prime}$ iff $n<n^{\prime}$, or $n=$ $n^{\prime}$ and $r_{1} \ldots r_{n} \preccurlyeq$ lex $r_{1}^{\prime} \ldots r_{n^{\prime}}^{\prime}$

Proposition 7. The ordering $\preccurlyeq$ set is a linear ordering on the set of knowledge bases over $N F C(\Sigma)$.

In the following, we will abbreviate $\mathcal{R} \prec_{\text {set }} \mathcal{R}^{\prime}$ simply by $\mathcal{R} \prec \mathcal{R}^{\prime}$, and analogously for the non-strict version $\preccurlyeq$ set .

Definition 7 ( $\rho$ NF (Beierle and Haldimann 2020a)). A knowledge base $\mathcal{R} \subseteq N F C(\Sigma)$ is in renaming normal form $(\rho \mathrm{NF})$ if for every $\mathcal{R}^{\prime}$ with $\mathcal{R} \simeq \mathcal{R}^{\prime}$ it holds that $\mathcal{R} \preccurlyeq \mathcal{R}^{\prime}$.

Note that we defined the $\rho \mathrm{NF}$ only for knowledge bases containing normal form conditionals; a generalized definition of the $\rho \mathrm{NF}$ for arbitrary conditionals is given in (Beierle and Haldimann 2020b). The $\rho$ NF can be combined with other normal forms. A knowledge base is in renaming reduced antecedent normal form ( $\rho \mathrm{RANF}$ ) if it is both in RANF and in $\rho \mathrm{NF}$, and it is in renaming antecedent normal form ( $\rho \mathrm{ANF}$ ) if it is both in $\rho \mathrm{NF}$ and in ANF. A list of knowledge bases in $\rho$ RANF can be generated by algorithm $K B^{\rho r a}$ (Beierle and Haldimann 2020a, Algorithm 2).

Proposition $8\left(K B^{\rho r a}\right)$. Applying $K B^{\rho r a}$ to an ordered signature $(\Sigma, \lessdot)$ terminates and returns a set of knowledge bases $\mathcal{K} \mathcal{B}$ such that:

( $\stackrel{p}{\sim}$ completeness) If $\mathcal{R}$ is a consistent $\Sigma$-knowledge base then there is $\mathcal{R}^{\prime} \in \mathcal{K B}$ and a signature renaming $\rho$ such that $\mathcal{R} \stackrel{p}{\sim} \rho\left(\mathcal{R}^{\prime}\right)$.

Proof. The proof follows from model completeness of $K B^{\text {Pra }}$ (Beierle and Haldimann 2020a, Prop. 11) and Proposition 2.

Observation 5 ( $\rho$ RANF). $K B^{\rho r a}$ generates systematically consistent knowledge bases in $\rho$ RANF over a given signature using only normal form conditionals. Our implementation of $K B^{\rho r a}$ shows that there are exactly $2.143 \Sigma_{a b^{-}}$ knowledge bases, with at most 4 conditionals, in $\rho$ RANF.

From the list of knowledge bases in $\rho$ RANF we can easily obtain the set of all knowledge bases in RANF. Algorithm $K B^{r a}$ (Algorithm 1) shows one way to do this.

Proposition $9\left(K B^{r a}\right)$. Algorithm 1 generates all knowledge bases in $R A N F$, i.e., applying $K B^{r a}$ to an ordered signature $(\Sigma, \lessdot)$ terminates and returns a set of knowledge bases $\mathcal{K} \mathcal{B}$ such that:

(RANF) All knowledge bases in $\mathcal{K B}$ are in $R A N F$. (RANF completeness) If a consistent knowledge base $\mathcal{R} \in$ $N F C(\Sigma)$ is in $R A N F$, then $\mathcal{R} \in \mathcal{K} \mathcal{B}$.

$(\stackrel{p}{\sim}$ completeness) $\quad$ If $\mathcal{R}$ is a consistent $\Sigma$-knowledge base then there is $\mathcal{R}^{\prime} \in \mathcal{K} \mathcal{B}$ such that $\mathcal{R} \stackrel{p}{\sim} \mathcal{R}^{\prime}$. 


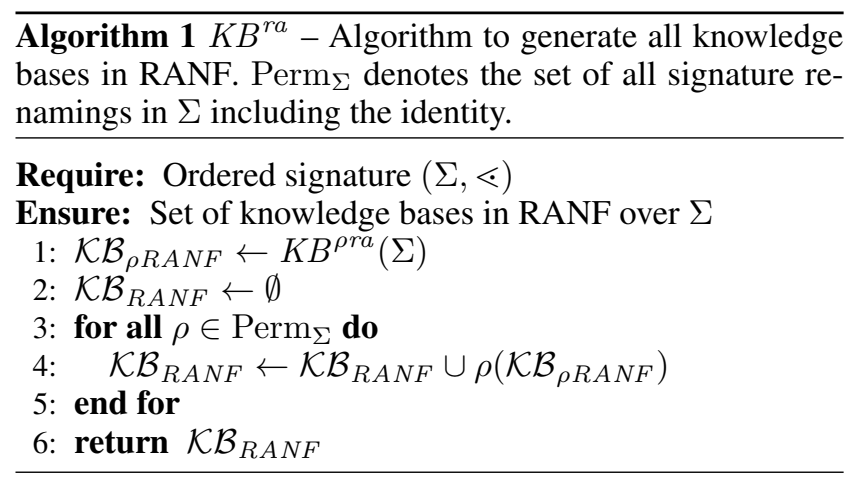

Proof. (RANF) All knowledge bases in $\mathcal{K} \mathcal{B}_{\rho R A N F}=$ $K B^{\rho r a}(\Sigma)$ are in RANF. Applying a renaming preserves the RANF. Therefore, all knowledge bases in $\mathcal{K B}$ are in RANF.

(RANF completeness) Let $\mathcal{R}$ be a knowledge base in RANF. Then there is a $\mathcal{R}^{\prime}$ in $\rho$ RANF and a renaming $\rho$ such that $\mathcal{R}^{\prime}=\rho(\mathcal{R})$. Proposition 8 states that $\mathcal{R} \in K B^{\rho r a}(\Sigma)$ because $\mathcal{R}$ is in $\rho$ RANF. As all renamings, including the inverse of $\rho$, are applied to $\mathcal{R}^{\prime}, \mathcal{R}$ is added to $\mathcal{K B}$.

$(\stackrel{p}{\sim}$ completeness $) \quad$ For every knowledge base $\mathcal{R}$ there is a $\mathcal{R}^{\prime}$ in RANF that is inferentially equivalent to $\mathcal{R}$. With (RANF completeness) it follows that $\mathcal{R}^{\prime} \in \mathcal{K} \mathcal{B}$.

Observation 6 (RANF). $K B^{r a}$ generates systematically consistent knowledge bases over a given signature in RANF using only normal form conditionals. Our implementation of $K B^{r a}$ shows that there are exactly 4.168 knowledge bases in RANF over $\Sigma_{a b}$, containing at most 4 conditionals.

\section{System P Inference Relations over $\Sigma_{a b}$-Knowledge Bases}

In the previous sections, we introduced different normal forms for conditional knowledge bases all of which respect inferential equivalence with respect to system $\mathrm{P}$. There are still different knowledge bases in RANF (or $\rho$ RANF) that are inferentially equivalent with respect to system $\mathrm{P}$ inference. In this section, we want to take this equivalence into account.

Proposition 10. For every system $P$ inference relation $\sim$ there is a conditional knowledge base $\mathcal{R}$ in $R A N F$ such that $\sim=\sim_{\mathcal{R}}^{p}$.

Proof. Let $\sim$ be a system $\mathrm{P}$ inference relation. The set $\mathcal{R}^{\prime}=\{(B \mid A) \in N F C(\Sigma) \mid A \sim B\}$ is a finite knowledge base. $\mathcal{R}^{\prime}$ is complete in the sense that we cannot derive any normal form conditional from $\mathcal{R}^{\prime}$ with system $\mathrm{P}$ that is not already in $\mathcal{R}^{\prime}$. Consider any conditional $(C \mid B)$. Let $\left(C^{\prime} \mid B^{\prime}\right)$ be the normal form conditional that is equivalent to $(C \mid B)$. All of the four statements $B \sim C, B^{\prime} \sim C^{\prime}$, $\left(C^{\prime} \mid B^{\prime}\right) \in \mathcal{R}^{\prime}$, and $B^{\prime} \sim_{\mathcal{R}^{\prime}}^{p} C^{\prime}$ are equivalent to each other. Therefore, $\sim=\sim_{\mathcal{R}^{\prime}}^{p}$.

There exists a knowledge base $\mathcal{R}$ in RANF, that is model equivalent to $\mathcal{R}^{\prime}$ (Beierle and Haldimann 2020a, Proposition 2 ). Because of Proposition $2, \mathcal{R}$ and $\mathcal{R}^{\prime}$ are inferentially equivalent and therefore $น=\sim_{\mathcal{R}^{\prime}}^{p}=\sim_{\mathcal{R}}^{p}$.
Based on the equivalence relation $\stackrel{p}{\sim}$ and the ordering $\preccurlyeq$ on knowledge bases, we can define a unique normal form for each equivalence class with respect to $\stackrel{p}{\sim}$.

Definition 8 (SPNF). A knowledge base $\mathcal{R}$ is in system $P$ normal form (SPNF) if $\mathcal{R}$ is in RANF and for every knowledge base $\mathcal{R}^{\prime} \subseteq N F C(\Sigma)$ in RANF with $\mathcal{R} \stackrel{p}{\sim} \mathcal{R}^{\prime}$ it holds that $\mathcal{R} \preccurlyeq \mathcal{R}^{\prime}$.

As there are 256 different conditionals in CDNF (including trivial conditionals), we store a list of 256 Booleans for every inference relation. This representation is suitable for every inference relation, that does not distinguish syntactic variants of the formulas in the query. To generate the desired list of inference relations, we begin with the set of all knowledge bases in RANF, as generated by $K B^{r a}$. Then we calculate the inference relation for each of these knowledge bases. After removing duplicates, this is the list of all inference relations.

Observation 7. Our implementation shows that there are exactly 484 different system $\mathrm{P}$ inference relations over $\Sigma_{a b}$, and the same number of $\Sigma_{a b}$-knowledge bases in SPNF.

Proposition 11. If a knowledge base $\mathcal{R}$ is in $S P N F$, then for every other consistent knowledge base $\mathcal{R}^{\prime} \subseteq N F C(\Sigma)$ with $\mathcal{R} \stackrel{p}{\sim} \mathcal{R}^{\prime}$ it holds that $\mathcal{R} \preccurlyeq \mathcal{R}^{\prime}$.

Proof. Let $\mathcal{R}$ be in SPNF. Assume, there is a consistent $\mathcal{R}^{\prime} \subseteq N F C(\Sigma)$ such that $\mathcal{R}^{\prime} \stackrel{p}{\sim} \mathcal{R}$ and $\mathcal{R}^{\prime} \prec \mathcal{R}$. Let $\mathcal{R}^{\prime \prime} \in \Theta^{r a}\left(\mathcal{R}^{\prime}\right)$ be a knowledge base in RANF that is equivalent to $\mathcal{R}^{\prime}$. Because $\Theta^{r a}$ can only reduce the number of conditionals in $\mathcal{R}^{\prime}$ (the knowledge base is consistent and contains only normal form conditionals), we have $\mathcal{R}^{\prime \prime} \preccurlyeq \mathcal{R}^{\prime} \prec \mathcal{R}$. Furthermore, $\mathcal{R}^{\prime \prime}$ is model equivalent and therefore inferentially equivalent (with respect to system $\mathrm{P}$ ) to $\mathcal{R}^{\prime}$. This is a contradiction to $\mathcal{R}$ being in SPNF.

Proposition 12. For every consistent $\mathcal{R} \subseteq N F C(\Sigma)$ there is a knowledge base $\mathcal{R}^{\prime}$ in SPNF with $\mathcal{R} \stackrel{p}{\sim} \mathcal{R}^{\prime}$.

Proof. Let $\mathcal{R} \subseteq N F C(\Sigma)$. Let $\mathcal{R}^{\prime \prime}$ be a knowledge base in RANF that is equivalent to $\mathcal{R}$. Therefore, the equivalence class $\left[\mathcal{R}^{\prime \prime}\right]_{\sim}$ is non-empty. Because there are only finitely many knowledge bases in RANF, there is a minimum $\mathcal{R}^{\prime}$ in $\left[\mathcal{R}^{\prime \prime}\right]_{\stackrel{p}{\sim}} \cdot \mathcal{R}^{\prime}$ is in SPNF and $\mathcal{R} \stackrel{p}{\sim} \mathcal{R}^{\prime}$.

Now we also want to take renamings into account.

Definition $9(\stackrel{p}{\simeq})$. Two knowledge bases $\mathcal{R}, \mathcal{R}^{\prime}$ are inferentially equivalent up to signature renamings with respect to system $P$ inference, denoted as $\mathcal{R} \stackrel{p}{\simeq} \mathcal{R}^{\prime}$, if there is a renaming $\rho$ such that $\rho\left(\sim_{\mathcal{R}}^{p}\right)=\sim_{\mathcal{R}^{\prime}}^{p}$.

Definition 10 ( $\rho$ SPNF). A knowledge base $\mathcal{R}$ is in renaming system $P$ normal form ( $\rho \mathrm{SPNF}$ ) if $\mathcal{R}$ is in RANF and for every other knowledge base $\mathcal{R}^{\prime} \subseteq N F C(\Sigma)$ in RANF with $\mathcal{R} \stackrel{p}{\simeq} \mathcal{R}^{\prime}$ it holds that $\mathcal{R} \preccurlyeq \mathcal{R}^{\prime}$.

Proposition 13. If a knowledge base $\mathcal{R}$ is in $\rho S P N F$, then for every other knowledge base $\mathcal{R}^{\prime} \subseteq N F C(\Sigma)$ with $\mathcal{R} \stackrel{p}{\simeq}$ $\mathcal{R}^{\prime}$ it holds that $\mathcal{R} \preccurlyeq \mathcal{R}^{\prime}$. 
Proof. Let $\mathcal{R}$ be in $\rho$ SPNF. Assume there is a consistent $\mathcal{R}^{\prime} \subseteq N F C(\Sigma)$ such that $\mathcal{R}^{\prime} \stackrel{p}{\simeq} \mathcal{R}$ and $\mathcal{R}^{\prime} \prec \mathcal{R}$. Let $\mathcal{R}^{\prime \prime} \in \Theta^{r a}\left(\mathcal{R}^{\prime}\right)$ be a knowledge base in RANF that is equivalent to $\mathcal{R}^{\prime}$. Because $\Theta^{r a}$ can only reduce the number of conditionals in $\mathcal{R}^{\prime}$ (the knowledge base is consistent and contains only normal form conditionals), we have $\mathcal{R}^{\prime \prime} \preccurlyeq \mathcal{R}^{\prime} \prec \mathcal{R}$. Furthermore, $\mathcal{R}^{\prime \prime}$ and $\mathcal{R}^{\prime}$ are model equivalent and due to (1) inferentially equivalent with respect to system P. Hence, $\mathcal{R}^{\prime \prime} \stackrel{p}{\simeq} \mathcal{R}^{\prime}$ and $\mathcal{R}^{\prime} \stackrel{p}{\simeq} \mathcal{R}$. This is a contradiction to $\mathcal{R}$ being in $\rho$ SPNF.

Proposition 14. For every system $P$ inference relation $\sim$ there is a conditional knowledge base $\mathcal{R}$ in $\rho S P N F$ and a renaming $\rho$ such that $\sim=\rho\left(\sim_{\mathcal{R}}^{p}\right)$.

Proof. Let $\sim$ be a system P inference relation. There is a knowledge base $\mathcal{R}^{\prime}$ in RANF such that $\sim=\sim_{\mathcal{R}^{\prime}}^{p}$ (see Proposition 10). Therefore, the equivalence class $\left[\mathcal{R}^{\prime}\right]_{p}$ is non-empty. Because there are only finitely many knowledge bases in RANF, there is a minimum $\mathcal{R}$ in $\left[\mathcal{R}^{\prime}\right]_{\underline{\underline{p}}} \cdot \mathcal{R}$ is in $\rho$ SPNF and because $\mathcal{R} \stackrel{p}{\simeq} \mathcal{R}^{\prime}$ there is a renaming $\rho$ such that $\rho\left(\sim_{\mathcal{R}}^{p}\right)=\sim_{\mathcal{R}^{\prime}}^{p}=\sim$.

Observation 8. Our implementation shows that, up to renamings of the underlying signature, there are exactly 262 different system P inference relations over $\Sigma_{a b}$, and correspondingly, $262 \Sigma_{a b}$-knowledge bases in $\rho$ SPNF.

We can also relate knowledge bases $\mathcal{R}, \mathcal{R}^{\prime}$ by their acceptance of so-called base conditionals, i.e., normal form conditionals $(B \mid A)$ with $|A|=2$ and $|B|=1$, inducing the relation $R \sim$ base $\mathcal{R}^{\prime}$ that holds if, for all base conditionals $(B \mid A)$, we have $A \sim_{\mathcal{R}}^{p} B$ iff $A \sim_{\mathcal{R}^{\prime}}^{p} B$. Obviously, $\sim_{\text {base }}$ is an equivalence relation, and $\stackrel{p}{\sim}$ is a refinement of $\sim_{\text {base }}$.

Observation 9. There are 219 equivalence classes with respect to $\sim_{\text {base }}$ over all knowledge bases in RANF over $\Sigma_{a b}$.

A base conditional $r=\left(\left\{\omega_{1}\right\} \mid\left\{\omega_{1}, \omega_{2}\right\}\right)$ encodes that $\omega_{1}$ is strictly more plausible than $\omega_{2}$. This means that $\kappa\left(\omega_{1}\right)<$ $\kappa\left(\omega_{2}\right)$ for all ranking models $\kappa$ of a knowledge base containing $r$. Note that the 219 different equivalence classes with respect to $\sim_{b a s e}$ coincide with the 219 different partial orders on the four worlds over $\Sigma_{a b}$.

\section{Conclusions and Further Work}

We introduced the normal forms SPNF and $\rho$ SPNF for conditional knowledge bases and developed a complete map of all $\Sigma_{a b}$-knowledge bases with respect to these and several other normal forms. For instance, there are only 262 $\Sigma_{a b}$-knowledge bases in $\rho$ SPNF, and each consistent $\Sigma_{a b^{-}}$ knowledge base has a unique $\rho$ SPNF. We are currently extending our investigations to signatures with more elements and by taking inference relations other than the induced system $\mathrm{P}$ inference relation into account. These other inference relations include system $\mathrm{Z}$ inference (Goldszmidt and Pearl 1996) and inference relations obtained from different inference modes with respect to various classes of models (Beierle et al. 2021).
Acknowledgments For empirical evaluations underlying specific results and numbers reported here, we thank our students Inga Feick, Frank Leuchtmann, Thomas Prinz, Martin Senser, Felix Weimer, and Jon Witte. This work was supported by DFG Grant BE 1700/9-1 awarded to C. Beierle.

\section{References}

[Adams 1975] Adams, E. W. 1975. The Logic of Conditionals: An Application of Probability to Deductive Logic. Synthese Library. Dordrecht, NL: Springer Science+Business Media.

[Beierle and Haldimann 2020a] Beierle, C., and Haldimann, J. 2020a. Normal forms of conditional knowledge bases respecting system P-entailments. In FoIKS 2020, volume 12012 of LNCS, 22-41. Springer.

[Beierle and Haldimann 2020b] Beierle, C., and Haldimann, J. 2020b. Transforming conditional knowledge bases into renaming normal form. In Proceedings FLAIRS 2020, 563-568. AAAI Press.

[Beierle and Kutsch 2019a] Beierle, C., and Kutsch, S. 2019a. On the antecedent normal form of conditional knowledge bases. In ECSQARU 2019, volume 11762 of LNAI, 175-186. Springer.

[Beierle and Kutsch 2019b] Beierle, C., and Kutsch, S. 2019b. Systematic generation of conditional knowledge bases up to renaming and equivalence. In JELIA 2019, volume 11468 of LNAI, 279-286. Springer.

[Beierle et al. 2021] Beierle, C.; Eichhorn, C.; Kern-Isberner, G.; and Kutsch, S. 2021. Properties and interrelationships of skeptical, weakly skeptical, and credulous inference induced by classes of minimal models. Artificial Intelligence 297. (in press, online 2 Mar 2021).

[Benferhat, Dubois, and Prade 1999] Benferhat, S.; Dubois, D.; and Prade, H. 1999. Possibilistic and standard probabilistic semantics of conditional knowledge bases. J. of Logic and Computation 9(6):873-895.

[Dubois and Prade 1994] Dubois, D., and Prade, H. 1994. Conditional objects as nonmonotonic consequence relationships. Special Issue on Conditional Event Algebra, IEEE Transactions on Systems, Man and Cybernetics 24(12):1724-1740.

[Goldszmidt and Pearl 1996] Goldszmidt, M., and Pearl, J. 1996. Qualitative probabilities for default reasoning, belief revision, and causal modeling. Artif. Intell. 84:57-112.

[Kern-Isberner 2001] Kern-Isberner, G. 2001. Conditionals in nonmonotonic reasoning and belief revision, volume 2087 of LNAI. Springer.

[Kraus, Lehmann, and Magidor 1990] Kraus, S.; Lehmann, D.; and Magidor, M. 1990. Nonmonotonic reasoning, preferential models and cumulative logics. Artificial Intelligence 44:167-207.

[Lehmann and Magidor 1992] Lehmann, D., and Magidor, M. 1992. What does a conditional knowledge base entail? Artificial Intelligence 55:1-60.

[Pearl 1988] Pearl, J. 1988. Probabilistic Reasoning in Intelligent Systems. San Mateo, Ca.: Morgan Kaufmann.

[Robinson and Voronkov 2001] Robinson, J. A., and Voronkov, A., eds. 2001. Handbook of Automated Reasoning (in 2 volumes). Elsevier and MIT Press.

[Robinson 1965] Robinson, J. A. 1965. A machine oriented logic based on the resolution principle. Journal of the ACM 12(1):23-41.

[Spohn 1988] Spohn, W. 1988. Ordinal conditional functions: a dynamic theory of epistemic states. In Harper, W., and Skyrms, B., eds., Causation in Decision, Belief Change, and Statistics, II. Kluwer Academic Publishers. 105-134. 